\title{
Editorials
}

\section{Anesthesia of the upper airway}

M

OST anesthesiologists, despite their level of clinical experience and expertise, would agree that managing a patient with a difficult airway is the most challenging task in anesthesia. The inability to provide ventilation and perform tracheal intubation is associated with major anesthesia complications and death. ${ }^{1}$ Although there is no formal consensus as to the ideal approach to the management of the difficult airways of these patients, most anesthesiologists believe that the safest method is to perform an awake tracheal intubation. It is our responsibility to teach our trainees the art of awake tracheal intubation using a variety of techniques. Equally important, we must also educate our trainees to be sensitive to our patient's needs since most patients consider the application of topical anesthesia and the placement of the tracheal tube while awake an unpleasant and frightening experience. To avoid a surgical airway, such as tracheostomy and laryngotomy, William MacEwen reported the first several successful awake orotracheal intubations in 1880 without topical anesthesia. ${ }^{2}$ In his report, he carefully described the reactions of a patient during the placement of the tracheal tube: "On its introduction there was evinced considerable excitement, accompanied by a spasmodic fit of coughing which lasted for about a couple of minutes. In order to gain the patient's confidence, he was asked to hold with his own hand the portion of the tube which projected from his mouth, and told that he was at liberty to withdraw it if he felt it necessary ..... half an hour afterward he withdrew the tube..." One hundred and twenty years later, with the advances in pharmacology and anesthetic techniques, it is unacceptable for patients to experience such anguish, discomfort, and anxiety. As anesthesiologists, we must ensure that these unpleasant experiences are minimized.

Effective surface mucosal anesthesia of the upper airway can be achieved by direct application of a local anesthetic. The choice of the local anesthetic largely depends on its availability, clinical efficacy, and safety. Currently, lidocaine and cocaine are probably the most commonly used local anesthetics for topical anesthesia. Cocaine compares favourably with lidocaine, because of its topical anesthetic and vasoconstricting properties, making it useful for nasotracheal intubation. However, cocaine should be used with caution because of its addictive potential and the possibility for abuse, as well as its inherent toxicity, particularly with higher concentrations. ${ }^{3-4}$

Many techniques have been described to apply or deposit local anesthetics to the upper airway. These include applying a gel or ointment, gargling a viscous solution, depositing local anesthetic droplets via an atomizer (or nebulizer), utilizing a transtracheal injection, or by direct deposit of a local anesthetic (sprayas-you-go) to the airway through a fibreoptic bronchoscope..$^{5-9}$ In this issue, Chung et al. described a simple technique to provide topical anesthesia to the upper airway by using aspiration of lidocaine. ${ }^{10}$ The technique involves instilling a lidocaine solution onto the back of the protruded tongue while the patient is lying supine and breathing through the mouth. Using a fibreoptic bronchoscope positioned in the pharynx of several volunteers, the authors observed the distribution of tinted lidocaine solution during the instillation, Initially, the lidocaine solution flowed around the valecula and the piriform fossa and then, eventually, was aspirated into the trachea. Using this technique, 38 of 39 patients (97\%) tolerated awake tracheal intubation using a fibreoptic bronchoscope without complications or side effects.

Although this simple and useful technique shows promising results, it should be emphasized that even careful surface mucosal anesthesia may be inadequate for awake tracheal intubation in some patients as the stretch receptors at the root of the tongue which cause the gag reflex are submucosal and are not easily blocked

Address correspondence to: Dr. Orlando R. Hung, Department of Anaesthesia, Queen Elizabeth II Health Sciences Centre, Victoria General Hospital, Halifax, Nova Scotia, B3H 2Y9 Canada. Phone: 902-473-7767; Fax: 902-423-9454; E-mail: hungorla@is.dal.ca 
following topical application of local anesthetics. This gag reflex can be minimized by using the nasal route of intubation or a supplemental block of the lingual branch of the glossopharyngeal nerve (IX). ${ }^{11}$ In some "sensitive" patients, or patients with copious secretions or blood in the upper airway, blockade of the internal branch of the recurrent laryngeal nerve or transtracheal instillation of local anesthetic through the crico-thyroid membrane may be necessary.

Although Chung $e t$ al. stated that the use of antisialogogues was not necessary using the aspiration technique in their study, effective surface mucosal anesthesia generally requires a dry mucous membrane. Copious secretions in the upper airway, particularly with chronic smokers, dilutes local anesthetics applied to the mucosa as well as forming a mechanical barrier between the anesthestic and mucosa. Antisialogogues, such as atropine and glycopyrrolate, are invaluable adjuncts that will improve the effectiveness of topically applied local anesthesia.

Irrespective of the topical anesthetic technique used, it is important that the patient undergoing awake tracheal intubation be sedated, comfortable. and experience minimal anxiety. It is equally important that the anxiolytic be carefully administered by titration to a desired effect so that a close communication between the anesthesiologist and the patient can be maintained during the awake tracheal intubation. Although a balance of comfort and excessive sedation is a challenge, it is critical to ensure ventilation and oxygenation while minimizing the risk. of aspirating stomach contents, particularly in patients who present with a full stomach. The risk of aspiration can be minimized by the administration of pharmacological agents, such as ranitidine, and metoclopramide, prior to intubation. In some situations, the avoidance of intense anesthesia below the vocal cords may add a margin of safety.

Successful awake tracheal intubation depends on many factors. These include patient selection and cooperation, the proper preparation of the patient and intubating equipment, suppression of the laryngeal and tracheal sensation by local anesthetics, an effective and safe intubating technique with a skillful operator, judicious use of pharmacological agents to achieve cooperation, and close communication with the patient during the procedure. In the case reported by MacEwen, to gain the patient's confidence and a sense of control, the patient was asked to hold the tracheal tube. Similarly, during awake tracheal intubation, it is necessary that we gain the patient's trust and co-operation with anxiety reduction techniques, such as constant reassurance, gentleness, and patience, in combination with the use of a judicious amount of anxiolytic agent during the procedure.

Is it possible to develop an ideal technique to achieve anesthesia for the upper airway? A number of studies compared the effectiveness of topical application, transtracheal application of local anesthesia, or nerve blocks to achieve anesthesia of the upper airway. ${ }^{12-14}$ There were little or no differences in effectiveness among these techniques or a combination of these techniques. It is clear that further studies will be required. Will the technique described by Chung $e t a l$. be the ideal technique? Possibly. However, it would be unduly optimistic to suggest that an ideal anesthetic technique for the upper airway can ever be developed. In my opinion, it is extremely difficult, if not impossible, to use only one specific anesthetic technique to meet the demands of a variety of patients who have a wide spectrum of anatomical variability, perception of discomfort, and expectations. The "ideal" anesthetic method should be the one that is simple, effective, safe and works best in the anesthesiologist's "educated" hands with the available resources. It may even be a combination of these techniques provided that it is performed with a careful preparation, patience, genteness, and above all, a close communication with the patient. The best technique is the one that is tailored to the individual needs of the patient.

\section{Anesthésie des voies aériennes supérieures}

La plupart des anesthésiologistes, quel que soit leur niveau d'expérience et de compétence, reconnaîtront que la prise en charge d'un patient chez qui l'intubation est difficile représente le plus grand défi de l'anesthésie. L'impossibilité de procéder à la ventilation et à une intubation endotrachéale est associée à des complications majeures et à la mort. ${ }^{1}$ Bien qu'on ne s'entende pas sur la méthode d'intubation idéale de ces patients, presque tous les anesthésiologistes croient que l'intubation vigile est la plus sûre. Nous sommes responsables d'enseigner l'intubation vigile à nos stagiaires, d'après diverses techniques. Il est tout aussi important de les éduquer à se préoccuper des besoins des patients dont la plupart voient l'application d'anesthésique topique et la mise en place vigile d'un tube endotrachéal comme une expérience désagréable et angoissante. William MacEwen cite les 
premières intubations orotrachéales vigiles réussies en 1880 sans anesthésie locale et réalisées pour éviter une intubation chirurgicale, comme la trachéotomie et la laryngotomie. ${ }^{2}$ Il décrit soigneusement les réactions d'un patient pendant la mise en place du tube trachéal : "Son introduction a provoqué une agitation manifeste, accompagnée d'une quinte de toux spasmodique qui a duré quelques minutes environ. Afin de gagner la confiance du patient, on lui a demandé de tenir dans sa main la partie du tube qui sortait de sa bouche, et on lui a dit qu'il était libre de le retirer s'il le jugeait nécessaire..... une demi-heure plus tard il a retiré le tube...» Cent vingt ans après, avec les progrès de la pharmacologie et des techniques anesthésiques, il est inacceptable que des patients vivent autant d'angoisse, d'inconfort et d'anxiété. Nous, anesthésiologistes, devons nous assurer que ces expériences désagréables soient réduites au minimum.

L'anesthésie efficace de la muqueuse superficielle des voies aériennes supérieures peut être obtenue par l'application directe d'un anesthésique local dont le choix dépend surtout de sa disponibilité, de son efficacité clinique et de son innocuité. Actuellement, la lidocaüne et la cocaüne sont sans doute les anesthésiques locaux le plus souvent utilisés pour l'anesthésie topique. La cocaïne se compare avantageusement à la lidocaïne à cause de ses propriétés vasoconstrictives et d'anesthésique topique, ce qui la rend utile pour l'intubation nasotrachéale. Toutefois, la cocaïne devrait être utilisée avec prudence, étant donné sa toxicité inhérente, surtout en grandes concentrations, et la possibilité d'y devenir dépendant et d'en abuser. ${ }^{3 \cdot 4}$

De nombreuses techniques de dépôt ou d'application d'anesthésique local sur les voies aériennes supérieures ont été décrites. Elles incluent l'application d'un gel ou d'un onguent, le gargarisme d'une solution visqueuse, le dépôt d'anesthésique local en fines gouttelettes au moyen d'un atomiseur (ou nébuliseur), l'injection transtrachéale ou le dépôt direct d'un anesthésique local (spray-as-you-go) sur les voies aériennes par un fibroscope bronchique. ${ }^{5-9}$ Dans le présent numéro, Chung et coll. décrivent une technique simple d'anesthésie locale des voies aériennes supérieures par aspiration de lidocaine. ${ }^{10} \mathrm{La}$ technique implique l'instillation d'une solution de lidocainne sur le dos de la langue en protrusion pendant que le patient est en décubitus dorsal et a une respiration buccale. Utilisant une fibroscope bronchique mis en place dans le pharynx de quelques volontaires, les auteurs ont observé la distribution de la solution de lidocaïne colorée pendant l'instillation. Initialement, la lidocaïne a circulé dans la fossette glosso-épiglottique et les gouttières pharyngo-laryngées pour être aspirée par la suite dans la trachée. En utilisant cette technique, 38 des 39 patients (97\%) ont toléré l'intubation endotrachéale vigile à l'aide d'un fibroscope bronchique, sans complications ou effets secondaires.

Bien que cette technique simple et utile offre des résultats prometteurs, on doit souligner que, même soigneusement réalisée, une anesthésie de surface de la muqueuse peut être insuffisante pour une intubation endotrachéale vigile chez certains patients, car les récepteurs de tension de la racine de la langue qui causent le réflexe laryngé (haut-le-cour) sont sousmuqueux et ne sont pas facilement bloqués par l'application topique d'anesthésiques locaux. Ce réflexe pharyngé peut être diminué par l'utilisation de l'intubation par voie nasale ou d'un bloc d'appoint de la branche linguale du nerf glossopharyngien (IX)." Chez certains patients "sensibles», ou chez des patients qui ont des sécrétions abondantes ou du sang dans les voies aériennes supérieures, le blocage de la branche interne du nerf laryngé récurrent ou l'instillation transtrachéale d'anesthésique local au travers de la membrane crico-thyroïdienne peuvent être nécessaires.

Même si Chung et coll. affirment que les antisialogogues n'ont pas été nécessaires au cours de leur étude, lors de l'emploi de la technique d'aspiration, l'anesthésie efficace de la muqueuse de surface exige généralement une membrane muqueuse sèche. D'abondantes sécrétions dans les voies aériennes supérieures, chez les fumeurs chroniques en particulier, diluent les anesthésiques locaux appliqués à la muqueuse tout en formant une barrière mécanique entre les anesthésiques et la muqueuse. Les antisialogogues, comme l'atropine et le glycopyrrolate, sont des auxiliaires de grande valeur qui peuvent améliorer l'efficacité de l'anesthésie local d'application topique.

Sans égard à la technique anesthésique topique utilisée, il est important que le patient qui subit une intubation endotrachéale vigile reçoive une sédation, soit confortable et vive le moins d'anxiété possible. Il est important également que l'anxiolytique soit administré avec soin suivant un dosage adapté à l'effet voulu de sorte qu'une étroite communication puisse être maintenue entre l'anesthésiologiste et le patient pendant l'intubation endotrachéale vigile. Quoique l'équilibre entre le confort et la sédation excessive soit un défi, il est impérieux d'assurer la ventilation et l'oxygénation tout en réduisant le risque d'aspiration du contenu gastrique, surtout chez les patients qui se présentent avec l'estomac plein. Le risque d'aspiration peut être diminué par l'administration de médicaments, comme la ranitidine et le métoclopramide, avant l'intubation. Dans certaines situations, éviter 
l'anesthésie intense sous les cordes vocales peut ajouter une marge de sécurité.

L'intubation endotrachéale réussie dépend de nombreux facteurs. Ils comprennent la sélection des patients et leur coopération, la préparation adéquate du patient et du matériel d'intubation, la suppression de la sensibilité laryngée et trachéale par des anesthésiques locaux, une technique d'intubation efficace et sûre faite par un opérateur qualifié, un usage judicieux de médicaments pour assurer la coopération et une communication étroite avec le patient pendant l'intervention. Dans le cas cité par MacEwen, on a demandé au patient de tenir le tube trachéal afin de lui donner confiance et l'impression d'exercer un certain contrôle. De même, pendant l'intubation endotrachéale vigile, il est nécessaire de s'assurer de la confiance et de la coopération du patient en utilisant des techniques de réduction de l'anxiété, comme l'encouragement constant, la douceur et la patience, en combinaison avec l'emploi d'une quantité suffisante d'anxiolytique pendant l'intervention.

Est-il possible d'élaborer une technique idéale d'anesthésie des voies aériennes supérieures ? Dans de multiples études, on a comparé l'efficacité de l'application topique, de l'application transtrachéale d'anesthésique local ou de blocages nerveux pour réaliser l'anesthésie des voies aériennes supérieures. ${ }^{12-14}$ Il y avait peu ou pas de différence d'efficacité entre ces techniques ou une combinaison de ces techniques. Il est clair que d'autres études sont nécessaires. Est-ce que la technique décrite par Chung et coll. serait la technique idéale?

C'est possible. Cependant, on serait trop optimiste de suggérer qu'une technique idéale d'anesthésie des voies respiratoires supérieures puisse être jamais mise au point. Selon nous, il est extrêmement difficile, voire impossible, d'utiliser une seule technique anesthésique spécifique qui réponde aux demandes variées des patients qui présentent une grande variabilité anatomique, des différences de perception de l'inconfort et des attentes diversifiées. La méthode anesthésique «idéale» devrait être simple, efficace, sûre et fonctionner le mieux possible entre les mains «expertes» des anesthésiologistes passédant les ressources disponibles. Ce pourrait être une combinaison de ces techniques pourvu qu'elle soit réalisée à la suite d'une préparation attentive, avec patience, douceur et, surtout, une étroite communication avec le patient. La meilleure technique est celle qui est adaptée aux besoins individuels du patient.

\section{References}

1 Caplan RA, Posner KL, Ward RJ, Cheney FW. Adverse respiratory events in anesthesia: a closed claims analysis. Anesthesiology 1990; 72: 828-33.
2 MacEwen W. Introduction of tracheal cubes by the mouth instead of performing tracheotomy or laryngotomy. BMJ 1880; 2: 122-4.

3 Teale $C$, Gornes PJ, Muers $M F$, et al. Local anesthesia for fiberoptic bronchoscopy: comparison between intrathecal cocaine and lignocaine. Resp Med 1990; 84: 407-8.

4 Lange RA, Cigarroa $R G$, Yancy CW Jr, et al. Cocaine induced coronary vasoconstriction. $N$ Engl J Med 1989; 321: 1557-62.

5 Haasio J, Jokinen T, Numminen $M$, Rosenberg $P H$. Topical anaesthesia of gingival mucosa by $5 \%$ eutectic mixture of lignocaine and prilocaine or by $10 \%$ lignocaine spray. Br J Oral Maxillofac Surg 1990; 28: 99-101.

6 Cobn AI, McGraw SR, King WH. Awake intubation of the adult trachea using the Bullard laryngoscope. Can J Anaesth 1995; 42: 246-8.

7 Webb AR, Woodhead MA, Dalton HR, Grigg JA, Millard FJC. Topical nasal anaesthesia for fibreoptic bronchoscopy: patients' preference for lignocaine gel. Thorax 1989; 44: 674-5.

8 Randell $T$, Yli-Hankala $A$, Valli $H$, Lindgren $L$. Topical anaesthesia of the nasal mucosa for fibreoptic airway endoscopy. Br J Anaesth 1992: 68: 164-7.

9 Ovassapian A, Krejcie TC, Yelich SJ, Dykes MHM. Awake fibreoptic intubation in the patient at high risk of aspiration. Br J Anaesth 1989; 62: 13-6.

10 Chung DC, Mainland P-A, Kong $A S$. Anesthesia of the airway by aspiration of lidocaine. Can J Anaesth 1999; 46: 215-19.

11 Mullin $V$. Topical and regional anesthesia of the upper airway. In: Norton ML (Ed.). Atlas of the Difficult Intubation, 2nd ed. St. Louis: Mosby Year-Book, 1996: 84-94.

12 Grabam DR, Hay JG, Clague J, Nisar M, Earis JE. Comparison of three different methods used to achieve local anesthesia for fiberoptic bronchoscopy. Chest 1992; 102: 704-7.

13 Bigeleisen PE, Schisler JQ Finucane BT. Plasma lidocaine levels following transtracheal injection during topical anaesthesia of the upper airway. Can J Anaesth 1988; 35: S95.

14 Webb AR, Fernando SSD, Dalton HR, Arrowsmith JE, Woodhead $M A$, Cummin AR. Local anaesthesia for fibreoptic bronchoscopy: transcricoid injection or the "spray as you go" technique? Thorax 1990; 45: 474-7. 